\title{
A Relativistic Version of the Two-Level Atom in the Rest-Frame Instant Form of Dynamics
}

\author{
David Alba \\ Dipartimento di Fisica \\ Universita' di Firenze \\ Polo Scientifico, via Sansone 1 \\ 50019 Sesto Fiorentino, Italy \\ E-mail alba@fi.infn.it \\ Horace W. Crater \\ The University of Tennessee Space Institute \\ Tullahoma, TN 37388 USA \\ E-mail: hcrater@utsi.edu \\ Luca Lusanna \\ Sezione INFN di Firenze \\ Polo Scientifico \\ Via Sansone 1 \\ 50019 Sesto Fiorentino (FI), Italy \\ E-mail: lusanna@fi.infn.it
}

\begin{abstract}
We define a relativistic version of the two-level atom, in which an extended atom is replaced by a point particle carrying suitable Grassmann variables for the description of the two-level structure and of the electric dipole. After studying the isolated system "atom plus the electro-magnetic field" in the electric-dipole representation as a parametrized Minkowski theory, we give its restriction to the inertial rest frame and the explicit form of the Poincaré generators. After quantization we get a two-level atom with a spin $1 / 2$ electric dipole and the relativistic generalization of the Hamiltonians of the Rabi and Jaynes-Cummings models.
\end{abstract}


November 10, 2018 


\section{INTRODUCTION}

The Rabi model [1] and its rotating-wave approximation or Jaynes-Cumming model [2] of two-level atoms are approximations for the interaction of atoms with a quantized mode of an optical cavity very useful in quantum optics and atomic physics. See Ref.[3] for an elementary introduction and the review in Ref.[4] for a detailed account of recent development.

This model like all atomic physics is an approximation to QED, in which the atoms are described as non-relativistic particles in quantum mechanics (QM) with a coupling to the electro-magnetic field of order $1 / c$. For all the applications in which the energies involved do not cross the threshold of pair production, this description with a fixed number of particles is enough. Therefore atomic physics is formulated in the absolute Euclidean 3-space and use Newton absolute time, namely it is formulated in Galilei space-time. The main drawback is that, due to the $1 / c$ coupling to the electro-magnetic field there is not a realization of the kinematical Galilei group connecting non-relativistic inertial frames. To get a relativistic description we must reformulate the theory in Minkowski space-time with a well defined realization of the kinematical Poincaré group connecting relativistic inertial frames. This would lead to relativistic atomic physics as the quantization of a fixed number of classical relativistic charged scalar (or spinning) particles interacting with the classical electro-magnetic field.

In Refs. [5, 6] (based on the previous results of Refs. [7, 8]) we gave a consistent relativistic formulation of atomic physics with an explicit construction of the Poincaré generators in the inertial rest-frame instant form of dynamics. In these papers we considered $\mathrm{N}$ charged positive energy scalar particles (with Grassmann-valued electric charges to regularize the selfenergies) interacting with the electro-magnetic field in the radiation gauge in the framework of parametrized Minkowski theories [9].

Parametrized Minkowski theories allows one to describe every isolated system having a Lagrangian description in special relativity in both inertial and non-inertial frames as shown in Refs. [10, 11]. This formulation is based on a metrology-oriented description of non-inertial frames obtained with the $3+1$ point of view and the use of observer-dependent Lorentz scalar radar 4-coordinates. Let us give the world-line $x^{\mu}(\tau)$ of an arbitrary timelike observer carrying a standard atomic clock: $\tau$ is an arbitrary monotonically increasing function of the proper time of this clock. Then we give an admissible $3+1$ splitting of Minkowski space-time, namely a nice foliation with space-like instantaneous 3-spaces $\Sigma_{\tau}$ : it is the mathematical idealization of a protocol for clock synchronization (all the clocks in the points of $\Sigma_{\tau}$ indicate the same time as the atomic clock of the observer). On each 3-space $\Sigma_{\tau}$ we choose curvilinear 3 -coordinates $\sigma^{r}$ having the observer as origin. These are the radar 4-coordinates $\sigma^{A}=\left(\tau ; \sigma^{r}\right)$. If $x^{\mu} \mapsto \sigma^{A}(x)$ is the coordinate transformation from the Cartesian 4-coordinates $x^{\mu}$ of a reference inertial observer to radar coordinates, its inverse $\sigma^{A} \mapsto x^{\mu}=z^{\mu}\left(\tau, \sigma^{r}\right)$ defines the embedding functions $z^{\mu}\left(\tau, \sigma^{r}\right)$ describing the 3-spaces $\Sigma_{\tau}$ as an embedded 3-manifold into Minkowski space-time. These embedding functions in turn induce a 4-metric on $\Sigma_{\tau}$ by the following functional: ${ }^{4} g_{A B}\left(\tau, \sigma^{r}\right)=\left[z_{A}^{\mu} \eta_{\mu \nu} z_{B}^{\nu}\right]\left(\tau, \sigma^{r}\right)$, where $z_{A}^{\mu}\left(\tau, \sigma^{r}\right)=\partial z^{\mu}\left(\tau, \sigma^{r}\right) / \partial \sigma^{A}$ are tetrads (with inverse tetrads $z_{\mu}^{A}\left(\tau, \sigma^{r}\right)$ ) and ${ }^{4} \eta_{\mu \nu}=$ $\epsilon(+---)$ is the flat metric $(\epsilon= \pm 1$ according to either the particle physics $\epsilon=1$ or the general relativity $\epsilon=-1$ convention). While the 4 -vectors $z_{r}^{\mu}\left(\tau, \sigma^{u}\right)$ are tangent to $\Sigma_{\tau}$, so that the unit normal $l^{\mu}\left(\tau, \sigma^{u}\right)$ is proportional to $\epsilon_{\alpha \beta \gamma}^{\mu}\left[z_{1}^{\alpha} z_{2}^{\beta} z_{3}^{\gamma}\right]\left(\tau, \sigma^{u}\right)$, we have $z_{\tau}^{\mu}\left(\tau, \sigma^{r}\right)=$ $\left[N l^{\mu}+N^{r} z_{r}^{\mu}\right]\left(\tau, \sigma^{r}\right)\left(N\left(\tau, \sigma^{r}\right)=\epsilon\left[z_{\tau}^{\mu} l_{\mu}\right]\left(\tau, \sigma^{r}\right)\right.$ and $N_{r}\left(\tau, \sigma^{r}\right)=-\epsilon g_{\tau r}\left(\tau, \sigma^{r}\right)$ are the lapse 
and shift functions).

The foliation is nice and admissible if it satisfies the conditions ${ }^{1}$ :

1) $N\left(\tau, \sigma^{r}\right)>0$ in every point of $\Sigma_{\tau}$ (the 3 -spaces never intersect, avoiding the coordinate singularity of Fermi coordinates);

2) $\epsilon^{4} g_{\tau \tau}\left(\tau, \sigma^{r}\right)>0$, so as to avoid the coordinate singularity of the rotating disk, and with the positive-definite 3 -metric ${ }^{3} g_{r s}\left(\tau, \sigma^{u}\right)=-\epsilon^{4} g_{r s}\left(\tau, \sigma^{u}\right)$ having three positive eigenvalues (these are the Møller conditions [10]);

3) all the 3-spaces $\Sigma_{\tau}$ must tend to the same space-like hyper-plane at spatial infinity (so that there are always asymptotic inertial observers to be identified with the fixed stars).

In the description of isolated systems (particles, strings, fields, fluids) admitting a Lagrangian formulation the matter variables are replaced with new ones knowing the 3-spaces $\Sigma_{\tau}$. For a relativistic particle with world-line $x^{\mu}(\tau)$ we must make a choice of its energy sign: then it will be described by 3-coordinates $\eta^{r}(\tau)$ defined by the intersection of the world-line with $\Sigma_{\tau}$ : $x^{\mu}(\tau)=z^{\mu}\left(\tau, \eta^{r}(\tau)\right)$. As opposed to all the previous approaches to relativistic mechanics, the dynamical configuration variables are the 3-coordinates $\eta_{i}^{r}(\tau)$ and not the world-lines $x_{i}^{\mu}(\tau)$ (to rebuild them in an arbitrary frame we need the embedding defining that frame [7]). For the electro-magnetic field the potential $\tilde{A}_{\mu}\left(x^{\alpha}\right)$ has to be replaced with $A_{A}(\tau, \vec{\sigma})=z_{A}^{\mu}(\tau, \vec{\sigma}) \tilde{A}_{\mu}\left(z^{\alpha}(\tau, \vec{\sigma})\right)$, whose associated field strength is $F_{A B}(\tau, \vec{\sigma})=\left(\partial_{A} A_{B}-\partial_{B} A_{A}\right)(\tau, \vec{\sigma})$ (from now on we will use the vector notation $\vec{\sigma}$ for the curvilinear 3-coordinates $\sigma^{r}$ for the sake of simplicity).

Then the matter Lagrangian is coupled to an external gravitational field and the external 4 -metric is replaced with the 4-metric $g_{A B}(\tau, \vec{\sigma})$ of an admissible $3+1$ splitting of Minkowski space-time. With this procedure we get a Lagrangian depending on the given matter and on the embedding $z^{\mu}(\tau, \vec{\sigma})$, which is invariant under frame-preserving diffeomorphisms. As a consequence, there are four first-class constraints (an analogue of the super-Hamiltonian and super-momentum constraints of canonical gravity) implying that the embeddings $z^{\mu}\left(\tau, \sigma^{r}\right)$ are gauge variables, so that all the admissible non-inertial or inertial frames are gauge equivalent, namely physics does not depend on the clock synchronization convention and on the choice of the 3-coordinates $\sigma^{r}$ : only the appearances of phenomena change (not their order) by changing the notion of instantaneous 3-space. Even if the gauge group is formed by the frame-preserving diffeomorphisms, the matter energy-momentum tensor allows the determination of the ten conserved Poincaré generators $P^{\mu}$ and $J^{\mu \nu}$ (assumed finite) of every configuration of the system.

If we restrict ourselves to inertial frames, we can define the inertial rest-frame instant form of dynamics for isolated systems by choosing the $3+1$ splitting corresponding to the intrinsic inertial rest frame of the isolated system centered on an inertial observer: the instantaneous 3-spaces, named Wigner 3-space due to the fact that the 3-vectors inside them are Wigner spin-1 3-vectors [8], are orthogonal to the conserved 4-momentum $P^{\mu}$ of the configuration. The embedding defining the inertial rest frame is

$$
z_{(W)}^{\mu}(\tau, \vec{\sigma})=x_{o}^{\mu}+\epsilon_{A}^{\mu}(\vec{h}) \sigma^{A},
$$

${ }^{1}$ These conditions imply that global rigid rotations are forbidden in relativistic theories $[10,11]$. 
where $\epsilon_{\tau}^{\mu}(\vec{h})=\frac{P^{\mu}}{\sqrt{\epsilon P^{2}}}=h^{\mu}=\left(\sqrt{1+\vec{h}^{2}} ; \vec{h}\right), \epsilon_{r}^{\mu}(\vec{h})=\left(-h_{r} ; \delta_{r}^{i}-\frac{h^{i} h_{r}}{1+\sqrt{1+\vec{h}^{2}}}\right)$ (see Ref.[5] for the notations; we have $\left.z_{(W) A}^{\mu}(\tau, \vec{\sigma})=\epsilon_{A}^{\mu}(\vec{h})\right)$. The tetrads $\epsilon_{A}^{\mu}(\vec{h})$ are the column of the standard Wigner boost for time-like orbits; the inverse tetrads are $\epsilon_{\mu}^{A}(\vec{h})$

This framework allows the description of atomic physics [5, 6], spinning particles [12], massless particles [13], open Nambu string [14]. Moreover it allows us to get a consistent definition of relativistic quantum mechanics and relativistic entanglement in the inertial rest-frame [15] with a preliminary extension to non-inertial frames [16, 17].

In Section 6 and 7 of Ref.[6] we studied the electric-dipole approximation and the transition to the relativistic electric-dipole representation for a system of two charged positiveenergy particles. After the separation of the center of mass ${ }^{2}$, the relative motion of the two particles gives rise to a relativistic bound state at the quantum level whose levels simulate the levels of an atom.

In this paper we will give a relativistic description of a two-level atom with an electric dipole, in which we approximate the bound state with its center of mass endowed with additional structures, described by suitable Grassmann variables, which must generate only a two-level structure (replacing the energy levels implied by the Schroedinger equation for the relative motion) and a two-level electric dipole (replacing the relative variable) after quantization.

Therefore we get a particular monopole-dipole description of the extended two-body system ${ }^{3}$, in which the point-like atom has:

a) a generalized dipole description of the two levels;

b) an electric dipole, which after quantization can assume two values like the spin of a Pauli particle;

c) and an optional spin dipole if the two-level atom is spinning (moreover, if the two-level is charged instead of being neutral as it is always assumed, the spin dipole would also behave as a magnetic dipole as shown in Ref.[12]).

We will give an action principle including also the dynamical electro-magnetic field (so that we have an isolated system), whose interaction with the point-like atom is given in the electric-dipole representation (it contains an interaction term $\vec{d} \cdot \vec{E}$ ) and not in the standard one (containing the interaction term $\frac{d \vec{x}(t)}{d t} \cdot \vec{A}(t, \vec{x}(t))$ ).

\footnotetext{
${ }^{2}$ See Refs.[5, 6, 10] for the problems connected with the relativistic collective variables for an extended system, i.e. the canonical non-covariant (Newton-Wigner) center of mass, the covariant non-canonical Fokker-Pryce center of inertia and the non-covariant non-canonical Møller center of energy. All of them collapse in Newton center of mass in the non-relativistic limit.

${ }^{3}$ See Section 5 of Ref.[6] for the standard multipolar expansion of the two-body system. It includes the monopole (the center of mass) and the spin dipole (the magnetic dipole), but not the electric dipole. Moreover it implies all the levels of the two-body system after quantization and not only two.
} 
The aim of the paper is not to contribute to the phenomenology of atomic physics, but only to build an explicit realization of the Poincaré generators of the relativistic two-level atom.

In order to facilitate the comparison of the various terms of our relativistic version with its nonrelativistic counterpart, we present here the nonrelativisitic Hamiltonian model for a point-like two-level atom interacting with a single mode electromagnetic field

$$
\begin{aligned}
H= & \frac{\vec{\kappa}^{2}}{2 m}+\frac{\hbar}{2} \hat{\Omega} \sigma_{3}+\hbar \omega\left(\hat{a}_{e m}^{\dagger} \hat{a}_{e m}+\frac{1}{2}\right) \\
& +(c \vec{d} \cdot \overrightarrow{\mathcal{E}}(\tau))\left(\sigma_{+}+\sigma_{-}\right)\left(\hat{a}_{e m}+\hat{a}_{e m}^{\dagger}\right)
\end{aligned}
$$

It consists of the kinetic energy operator $\vec{\kappa}^{2} / 2 m$ of the center of mass motion, the Hamiltonian $\hbar \omega\left(\hat{a}_{e m}^{\dagger} \hat{a}_{e m}+\frac{1}{2}\right)$ of the free field and the Hamiltonian $\frac{\hbar}{2} \hat{\Omega} \sigma_{3}$ corresponding to the two-internal states of the atom. The final term corresponds to the interaction between these degrees of freedom. Even though there are no two-level atoms in nature, one can achieve a configuration in which only, in effect, two-levels are involved by optical pumping. It is our aim to not only obtain the relativistic quantum Dirac Hamiltonian corresponding to this model but also an explicit realization of the other Poincare generators, particularly the interaction dependent boost. In [4] and [18] the details are presented of a microsopic derivation of Eq. (1.2) for a hydrogen atom in a time-varying electro-magnetic field. We will not be presenting the microscopic derivation of the relativisitic version ${ }^{4}$. Rather we will be arriving at our goal by first constructing the (pseudo)-classical Lagrangian description of a point-like two-level atom as a parametrized Minkowski theory. This is done in Section II.

In Section III we study the constraints present in the Hamiltonian description and then we give the restriction of the isolated system to the inertial rest frame and the explicit form of the Poincaré generators.

In Section IV we show how the quantization of the Grassmann variables and of the constraints implies the two-level structure, a two-level electric dipole and the Hamiltonian of the Rabi model.

Some final comments are done in the Conclusions.

\footnotetext{
${ }^{4}$ In [11] we presented the classical version of this microscopic derivation including the presentation of the Poincaire generators.
} 


\section{THE LAGRANGIAN DESCRIPTION OF THE TWO-LEVEL ATOM IN A DY- NAMICAL ELECTRO-MAGNETIC FIELD}

Let us consider the isolated system of a two-level atom interacting with a dynamical electro-magnetic field in the framework of parametrized Minkowski theories..

The point-like two-level atom is described by the 3-coordinates $\eta^{r}(\tau)$, so that its worldline is given by $x^{\mu}(\tau)=z^{\mu}\left(\tau, \eta^{r}(\tau)\right)$ as said in the Introduction. This is the monopole description of an extended atom.

The monopole carries two pairs of complex Grassmann variables $\alpha(\tau), \alpha^{*}(\tau), \beta(\tau), \beta^{*}(\tau)$ needed for the description of a structure with only two energy levels. They satisfy $\alpha^{2}(\tau)=$ $\left(\alpha^{*}(\tau)\right)^{2}=\alpha(\tau) \alpha^{*}(\tau)+\alpha^{*}(\tau) \alpha(\tau)=0, \beta^{2}(\tau)=\left(\beta^{*}(\tau)\right)^{2}=\beta(\tau) \beta^{*}(\tau)+\beta^{*}(\tau) \beta(\tau)=0$, $\alpha(\tau) \beta(\tau)-\beta(\tau) \alpha(\tau)=\alpha(\tau) \beta^{*}(\tau)-\beta^{*}(\tau) \alpha(\tau)=\alpha^{*}(\tau) \beta(\tau)-\beta(\tau) \alpha^{*}(\tau)=\alpha^{*}(\tau) \beta^{*}(\tau)-$ $\beta^{*}(\tau) \alpha^{*}(\tau)=0$. As shown in Ref.[12] the quantization of each pair of complex Grassmann variables generates a two-level Fermi oscillator. These variables thus underly the quantum operators corresponding to the Pauli-matrices in Eq. (1.2)

To describe the electric dipole carried by the atom we use a real Grassmann 4-vector $\xi^{\mu}(\tau), \xi^{\mu}(\tau) \xi^{\nu}(\tau)+\xi^{\nu}(\tau) \xi^{\mu}(\tau)=0$, commuting with the Grassmann variables describing the two-level structure. To describe a two-level structure after quantization (like a spin 1/2 Pauli particle) it must satisfy the constraint $P_{\mu} \xi^{\mu} \approx 0$, where $P^{\mu}$ is the total conserved 4 -momentum of the isolated system. Since, as shown in Ref.[12], it is too difficult to include this condition with a Lagrange multiplier in the Lagrangian, we will give a Lagrangian not implying this condition. Instead we will add it by hand in the resulting Hamiltonian description. Then conceivably one could try to derive the correct Lagrangian by means of the inverse Legendre transformation. The variables $\xi^{\mu}(\tau)$ allow us to build a spin-like tensor $S^{\mu \nu}(\tau)=-i \xi^{\mu}(\tau) \xi^{\nu}(\tau)$ satisfying $P_{\mu} S^{\mu \nu}(\tau) \approx 0$. In the Wigner 3-spaces of the rest frame the constraint $P_{\mu} \xi^{\mu} \approx 0$ implies that only the following three Grassmann variables survive

$$
\xi_{\perp}^{r}(\tau) \stackrel{\text { def }}{=} \epsilon_{\mu}^{r}(\vec{h}) \xi^{\mu}(\tau) .
$$

Then the rest-frame spin-like tensor $\bar{S}^{A B}(\tau)=\epsilon_{\mu}^{A}(\vec{h}) \epsilon_{\nu}^{B}(\vec{h}) S^{\mu \nu}(\tau)$ satisfies $\bar{S}^{\tau B}(\tau) \approx 0$ and $\bar{S}^{r}(\tau)=\frac{1}{2} \epsilon^{r u v} \bar{S}^{u v}(\tau)=-\frac{i}{2} \epsilon^{r u v} \xi_{\perp}^{u}(\tau) \xi_{\perp}^{v}(\tau)$. As shown in Ref.[12] the quantization of the three real Grassmann variables $\xi_{\perp}^{r}$ generates the algebra of Pauli matrices $\left(\xi_{\perp}^{r} \mapsto \sqrt{\frac{\hbar}{2}} \sigma^{r}\right)$ describing the $\left(\frac{1}{2}, 0\right)$ representation of $\mathrm{SL}(2, \mathrm{C})$. They will be used in the construction of the dipole operator $\vec{d}$ in Eq. (1.2)

In this paper we consider only the electric dipole. If the two-level atom is charged, we could also introduce the magnetic dipole by adding a spin dipole described by a real Grassmann 4-vector $\xi_{Q}^{\mu}(\tau)$ satisfying $P_{\mu} \xi_{Q}^{\mu}(\tau) \approx 0$ as done in Ref.[12] to describe a positive energy spinning particle giving rise to a positive energy spin $1 / 2$ particle at the quantum level. In this case we need also a Grassmann-valued electric charge $Q(\tau)=\theta^{*}(\tau) \theta(\tau)$ described by a pair of complex Grassmann variables (it turns out to be a constant of motion, $\frac{d Q(\tau)}{d \tau}=0$ ). Again each type of Grassmann variable is commuting with the Grassmann variables of a different type.

In Ref.[12] the mass term in the Lagrangian for the spinning particle was 


$$
\begin{aligned}
& \sqrt{m^{2} c^{2}+Q S_{Q}^{\mu \nu}(\tau) F_{\mu \nu}(z(\tau, \vec{\sigma}))}= \\
& =\sqrt{m^{2} c^{2}-i Q \xi_{Q}^{\mu}(\tau) \xi_{Q}^{\nu} z_{\mu}^{A}(\tau, \vec{\sigma}) z_{\nu}^{B}(\tau, \vec{\sigma}) F_{A B}(\tau, \vec{\sigma})} \approx \\
& \approx \sqrt{m^{2} c^{2}-2 Q \vec{S}_{Q} \cdot \vec{B}(\tau, \vec{\sigma})}= \\
& =m c-Q \vec{S}_{Q} \cdot \vec{B}(\tau, \vec{\sigma}),
\end{aligned}
$$

where we used the implications of the constraint $P_{\mu} \xi_{Q}^{\mu}(\tau) \approx 0$ on the spin tensor $S_{Q}^{\mu \nu}=$ $-i \xi_{Q}^{\mu}(\tau) \xi_{Q}^{\nu}(\tau)$ to show the emergence of the coupling of the spin dipole with the magnetic field.

By analogy we get the coupling of the electric dipole, described by the Grassmann variables $\xi^{\mu}(\tau)$, to the electric field by modifying Eq.(2.2) through the introduction of a coupling of the associated spin-like term $S_{\xi}^{\mu \nu}(\tau)=-i \xi^{\mu}(\tau) \xi^{\nu}(\tau)$ with the dual $F_{\mu \nu}^{*}=\frac{1}{2} \epsilon_{\mu \nu \alpha \beta} F^{\alpha \beta}$ of the field strength: now $S^{\mu \nu} F_{\mu \nu}^{*}=-\frac{i}{2} \xi^{\mu} \xi^{\nu} \epsilon_{\mu \nu}^{\alpha \beta} z_{\alpha}^{A} z_{\beta}^{B} F_{A B}$ will become $\vec{S} \cdot \vec{E}$ which will correspond to the electric-dipole coupling.

The previous discussion and the form of the Hamiltonian given in Eq. (1.2) of the JaynesCummings model suggests replacing the mass $m c$ with the following expression

$$
\begin{aligned}
m^{*}(\tau, \vec{\eta}(\tau)) c= & m c+\Omega \beta^{*}(\tau) \beta(\tau)+ \\
+ & \frac{i}{2} d\left(\beta^{*}(\tau) \alpha(\tau)+\alpha^{*}(\tau) \beta(\tau)\right) \xi^{\mu}(\tau) \xi^{\nu}(\tau) \epsilon_{\mu \nu}{ }^{\alpha \beta}\left(z_{\alpha}^{A}(\tau, \vec{\sigma}) z_{\beta}^{B}(\tau, \vec{\sigma})\right. \\
& \left.\times F_{A B}(\tau, \vec{\sigma})\right)\left.\right|_{\vec{\sigma}=\vec{\eta}(\tau)} .
\end{aligned}
$$

Here $\Omega$ is the energy difference between the two levels and $d$ is the coupling constant between the electric dipole and the electric field.

Therefore in the framework of parametrized Minkowski theories our isolated system is described:

a) by the 3-coordinates $\eta^{r}(\tau)$ of the point-like atom;

b) by two pairs of complex Grassmann variables $\alpha(\tau)$ and $\beta(\tau)$ describing the levels of the atom;

c) by a real Grassmann 4-vector $\xi^{\mu}(\tau)$ (to be reduced to a Wigner spin-1 3-vector $\xi_{\perp}^{r}(\tau)$ at the Hamiltonian level with the addition of the constraint $\left.P_{\mu} \xi^{\mu}(\tau) \approx 0\right)$ to describe the electric dipole;

d) by the electro-magnetic potential $A_{A}(\tau, \vec{\sigma})$ defined in the Introduction; 
e) by the embedding $z^{\mu}(\tau, \vec{\sigma})$ describing the $3+1$ splitting, which will be restricted to the embedding of the Wigner 3-spaces of the inertial rest frame at the Hamiltonian level at the end.

The Lagrangian of the system, describing it as a parametrized Minkowski theory, is $\left(\dot{a}(\tau)=\frac{d a(\tau)}{d \tau} ; m^{*}(\tau, \vec{\sigma})\right.$ given in Eq. $\left.(2.3)\right)$

$$
\begin{aligned}
L(\tau)= & \int d^{3} \sigma \mathcal{L}(\tau, \vec{\sigma}) \\
\mathcal{L}(\tau, \vec{\sigma})= & \delta^{3}(\vec{\sigma}-\vec{\eta}(\tau)) \\
& {\left[\frac{i}{2}\left(\xi_{\mu}(\tau) \dot{\xi}^{\mu}(\tau)+\beta^{*}(\tau) \dot{\beta}(\tau)-\dot{\beta}^{*}(\tau) \beta(\tau)+\alpha^{*}(\tau) \dot{\alpha}(\tau)-\dot{\alpha}^{*}(\tau) \alpha(\tau)\right)-\right.} \\
- & m^{*}(\tau, \vec{\sigma}) c \sqrt{{ }^{4} g_{\tau \tau}+2^{4} g_{\tau r} \dot{\eta}^{r}(\tau)+{ }^{4} g_{r s} \dot{\eta}^{r}(\tau) \dot{\eta}^{s}(\tau)}(\tau, \vec{\sigma})+ \\
+ & \left.\lambda_{1}(\tau)\left(\alpha^{*}(\tau) \alpha(\tau)+\beta^{*}(\tau) \beta(\tau)\right)\right]- \\
- & \frac{1}{4}\left(\sqrt{\left|{ }^{4} g\right|}{ }^{4} g^{A C} g^{B D} F_{A B} F_{C D}\right)(\tau, \vec{\sigma})
\end{aligned}
$$

Its construction will ultimately allow us to obtain expressions for the Poincaire generators of Eq. (3.17). The first term is the kinetic term for the Grassmann variables. The Lagrange multiplier $\lambda_{1}(\tau)$ is needed to get a constraint on the Grassmann variables which reduces the levels of the atom from four to two and is considered as a configuration variable.

Let us remark that the analogous Lagrangian for the spinning particle of Ref. [12]: 1) does not contains the variables $\alpha(\tau)$ and $\beta(\tau)$ (but has the Grassmann variables for the electric charge $\left.Q=\theta^{*}(\tau) \theta(\tau)\right)$; 2) has $m c+\Omega \beta^{*}(\tau) \beta(\tau)$ replaced by the ordinary mass term $m c$; 3) has the electric-dipole term $\frac{i}{2} d\left(\beta^{*}(\tau) \alpha(\tau)+\alpha^{*}(\tau) \beta(\tau)\right) \xi^{\mu}(\tau) \xi^{\nu}(\tau) \epsilon_{\mu \nu}{ }^{\alpha \beta}$ replaced by the spin-dipole term $-\frac{i}{2 m c} Q \xi_{Q}^{\alpha}(\tau) \xi_{Q}^{\beta}(\tau)$; 4) has the minimal coupling term $\delta^{3}(\vec{\sigma}-\vec{\eta}(\tau)) Q\left(A_{\tau}(\tau, \vec{\sigma})+\dot{\eta}^{r}(\tau) A_{r}(\tau, \vec{\sigma})\right)$ not being in the electric dipole representation. If we would consider a charged and spinning two-level atom, instead of the standard neutral one, we should add terms like in items 1), 3) and 4) to the Lagrangian (2.4).

The canonical momenta associated with the Grassmann variables and with the Lagrangian multiplier are

$$
\begin{array}{rlrl}
\pi_{\xi}^{\mu}(\tau) & =\frac{i}{2} \xi^{\mu}(\tau), & & \\
\pi_{\alpha}(\tau) & =-\frac{i}{2} \alpha^{*}(\tau), & \pi_{\alpha}^{*}(\tau)=+\frac{i}{2} \alpha(\tau), \\
\pi_{\beta}(\tau) & =-\frac{i}{2} \beta^{*}(\tau), & \pi_{\beta}^{*}(\tau)=+\frac{i}{2} \beta(\tau), \\
\pi_{\lambda_{1}}(\tau) & =0
\end{array}
$$

If we introduce the following notation for the electric dipole 


$$
A^{\alpha \beta}(\tau)=\frac{i}{2} d\left(\beta^{*}(\tau) \alpha(\tau)+\alpha^{*}(\tau) \beta(\tau)\right) \xi^{\mu}(\tau) \xi^{\nu}(\tau) \epsilon_{\mu \nu}^{\alpha \beta}
$$

we have that the terms bilinear in this quantity vanish because we have $\left(\beta^{*}(\tau) \alpha(\tau)+\right.$ $\left.\alpha^{*}(\tau) \beta(\tau)\right)^{2}=0$ due to the properties of the Grassmann variables.

Then the canonical momenta associated with the atom 3-position and with the electromagnetic field have the following forms

$$
\begin{aligned}
\kappa_{r}(\tau)= & -\frac{\partial L(\tau)}{\partial \dot{\eta}^{r}}=\left(m c+\Omega \beta^{*}(\tau) \beta(\tau)+\right. \\
+ & \left.A^{\alpha \beta}(\tau) z_{\alpha}^{A}(\tau, \vec{\eta}(\tau)) z_{\beta}^{B}(\tau, \vec{\eta}(\tau)) F_{A B}(\tau, \vec{\eta}(\tau))\right) \\
& \frac{{ }^{4} g_{\tau r}(\tau, \vec{\eta}(\tau))+{ }^{4} g_{r s}(\tau, \vec{\eta}(\tau)) \dot{\eta}^{r}(\tau)}{\sqrt{{ }^{4} g_{\tau \tau}(\tau, \vec{\eta}(\tau))+2^{4} g_{\tau u}(\tau, \vec{\eta}(\tau)) \dot{\eta}^{u}(\tau)+{ }^{4} g_{u v}(\tau, \vec{\eta}(\tau)) \dot{\eta}^{u}(\tau) \dot{\eta}^{v}(\tau)}} \\
\pi^{\tau}(\tau, \vec{\sigma})= & \frac{\partial \mathcal{L}(\tau, \vec{\sigma})}{\partial\left(\partial_{\tau} A_{\tau}\right)}=0, \\
\pi^{r}(\tau, \vec{\sigma})= & \frac{\partial \mathcal{L}(\tau, \vec{\sigma})}{\partial\left(\partial_{\tau} A_{r}\right)}=-\left[\frac{\gamma}{\sqrt{{ }^{4} g}} \gamma^{r s}\left(F_{\tau s}-{ }^{4} g_{\tau v} \gamma^{u v} F_{u s}\right)\right](\tau, \vec{\sigma})- \\
- & \delta^{3}(\vec{\sigma}-\vec{\eta}(\tau)) \frac{2\left(m c+\Omega \beta^{*}(\tau) \beta(\tau)\right) A^{\alpha \beta}(\tau)\left(l_{\alpha} z_{s \beta} \gamma^{s r}\right)(\tau, \vec{\sigma})}{\sqrt{m^{2} c^{2}+2 m c \Omega \beta^{*}(\tau) \beta(\tau)+\gamma^{u v}(\tau, \vec{\sigma}) \kappa_{u}(\tau) \kappa_{v}(\tau)}}
\end{aligned}
$$

where $\gamma^{r s}(\tau, \vec{\sigma})$ is the inverse of the positive-signature 3 -metric ${ }^{3} g_{r s}(\tau, \vec{\sigma})=-\epsilon^{4} g_{r s}(\tau, \vec{\sigma})$, $\gamma(\tau, \vec{\sigma})=\operatorname{det}^{3} g_{r s}(\tau, \vec{\sigma}),{ }^{4} g(\tau, \vec{\sigma})=\left|\operatorname{det}^{4} g_{A B}(\tau, \vec{\sigma})\right|$ and $l^{\alpha}(\tau, \vec{\sigma})$ is the unit normal to the 3 -space $\Sigma_{\tau}$ in the point with 3 -coordinates $\vec{\sigma}$.

Finally the canonical momentum conjugate to the embedding $z^{\mu}(\tau, \vec{\sigma})$ is 


$$
\begin{aligned}
& \rho_{\mu}(\tau, \vec{\sigma})=-\frac{\partial \mathcal{L}(\tau, \vec{\sigma})}{\partial z_{\tau}^{\mu}}=\delta^{3}(\vec{\sigma}-\vec{\eta}(\tau)) \\
& {\left[\left(m c+\Omega \beta^{*}(\tau) \beta(\tau)+A^{\alpha \beta}(\tau) z_{\alpha}^{A}(\tau, \vec{\sigma}) z_{\beta}^{B}(\tau, \vec{\sigma}) F_{A B}(\tau, \vec{\sigma})\right)\right.} \\
& \frac{z_{\tau \mu}(\tau, \vec{\sigma})+z_{r \mu}(\tau, \vec{\sigma}) \dot{\eta}^{r}(\tau)}{\sqrt{{ }^{4} g_{\tau \tau}(\tau, \vec{\sigma})+2{ }^{4} g_{\tau u}(\tau, \vec{\sigma}) \dot{\eta}^{u}(\tau)+{ }^{4} g_{u v}(\tau, \vec{\sigma}) \dot{\eta}^{u}(\tau) \dot{\eta}^{v}(\tau)}}+ \\
& -2 A^{\rho \sigma}(\tau)\left[z_{\tau \mu}\left({ }^{4} g^{A \tau 4} g^{\tau C 4} g^{B D}+{ }^{4} g^{A C 4} g^{B \tau}{ }^{4} g^{\tau D}\right)+\right. \\
& \left.+z_{r \mu}\left({ }^{4} g^{A r 4} g^{\tau C}+{ }^{4} g^{A \tau}{ }^{4} g^{r C}\right)^{4} g^{B D}\right](\tau, \vec{\sigma}) \times \\
& \times\left(z_{C \rho} z_{D \nu} F_{A B}\right)(\tau, \vec{\sigma}) \sqrt{{ }^{4} g_{\tau \tau}(\tau, \vec{\sigma})+2{ }^{4} g_{\tau u}(\tau, \vec{\sigma}) \dot{\eta}^{u}(\tau)+{ }^{4} g_{u v}(\tau, \vec{\sigma}) \dot{\eta}^{u}(\tau) \dot{\eta}^{v}(\tau)}+ \\
& -2 A_{\mu \rho}(\tau)\left({ }^{4} g^{A \tau}{ }^{4} g^{B D} z_{D}^{\rho} F_{A B}\right)(\tau, \vec{\sigma}) \\
& \left.\sqrt{{ }^{4} g_{\tau \tau}(\tau, \vec{\sigma})+2{ }^{4} g_{\tau u}(\tau, \vec{\sigma}) \dot{\eta}^{u}(\tau)+{ }^{4} g_{u v}(\tau, \vec{\sigma}) \dot{\eta}^{u}(\tau) \dot{\eta}^{v}(\tau)}\right]+ \\
& +\frac{\sqrt{{ }^{4} g}}{4}\left[{ }^{4} g^{\tau E} z_{E \mu}{ }^{4} g^{A C 4} g^{B D} F_{A B} F_{C D}-2 z_{\tau \mu}\left({ }^{4} g^{A \tau} 4 g^{\tau C}{ }^{4} g^{B D}+{ }^{4} g^{A C}{ }^{4} g^{B \tau}{ }^{4} g^{\tau D}\right) F_{A B} F_{C D}+\right. \\
& \left.-2 z_{r \mu}\left({ }^{4} g^{A r 4} g^{\tau C}+{ }^{4} g^{A \tau}{ }^{4} g^{r C}\right)^{4} g^{B D} F_{A B} F_{C D}\right](\tau, \vec{\sigma}) \text {. }
\end{aligned}
$$

The canonical Hamiltonian is

$$
H_{c}=-\lambda_{1}(\tau)\left(\alpha^{*}(\tau) \alpha(\tau)+\beta^{*}(\tau) \beta(\tau)\right)-\int d^{3} \sigma A_{\tau}(\tau, \vec{\sigma}) \partial_{r} \pi^{r}(\tau, \vec{\sigma}) .
$$

while the conserved Poincaré generators are (see Ref. $[9,12]$ )

$$
\begin{aligned}
P^{\mu} & =\int d^{3} \sigma \rho^{\mu}(\tau, \vec{\sigma}) \\
J^{\mu \nu} & =\int d^{3} \sigma\left[z^{\mu}(\tau, \vec{\sigma}) \rho^{\nu}(\tau, \vec{\sigma})-z^{\nu}(\tau, \vec{\sigma}) \rho^{\mu}(\tau, \vec{\sigma})\right]+i \xi^{\mu}(\tau) \xi^{\nu}(\tau) .
\end{aligned}
$$




\section{THE HAMILTONIAN DESCRIPTION}

Let us now describe the primary Dirac constraints of the model.

The Grassmann momenta (2.5) imply the following second class constraints

$$
\begin{aligned}
& \chi^{\mu}(\tau)=\pi_{\xi}^{\mu}(\tau)-\frac{i}{2} \xi^{\mu}(\tau) \approx 0 \\
& \chi_{(\beta)}(\tau)=\pi_{\beta}(\tau)+\frac{i}{2} \beta^{*}(\tau) \approx 0, \quad \chi_{(\beta)}^{*}(\tau)=\pi_{\beta}^{*}(\tau)-\frac{i}{2} \beta(\tau) \approx 0, \\
& \chi_{(\alpha)}(\tau)=\pi_{\alpha}(\tau)+\frac{i}{2} \alpha^{*}(\tau) \approx 0, \quad \chi_{(\alpha)}^{*}(\tau)=\pi_{\alpha}^{*}(\tau)-\frac{i}{2} \alpha(\tau) \approx 0 .
\end{aligned}
$$

The Poisson brackets of the Grassmann variables are $\left\{\xi^{\mu}(\tau), \pi_{\xi}^{\nu}(\tau)\right\}=-\eta^{\mu \nu}$, $\left\{\alpha(\tau), \pi_{\alpha}(\tau)\right\}=\left\{\alpha^{*}(\tau), \pi_{\alpha}^{*}(\tau)\right\}=\left\{\beta(\tau), \pi_{\beta}(\tau)\right\}=\left\{\beta^{*}(\tau), \pi_{\beta}^{*}(\tau)\right\}=-1$.

The other primary constraints are

$$
\begin{aligned}
\pi_{\lambda_{1}}(\tau) & \approx 0, \\
\pi^{\tau}(\tau, \vec{\sigma}) & \approx 0, \\
\mathcal{H}_{\mu}(\tau, \vec{\sigma}) & \approx 0,
\end{aligned}
$$

where, following the methods of Ref.[12] and using the results given after Eq.(2.6), one has the following final expression for the constraints $\mathcal{H}_{\mu}(\tau, \vec{\sigma}) \approx 0$ deriving from Eqs.(2.8) (they imply that the embeddings are gauge variables or equivalently, they provide a four-vector continuum set of first class constraints associated with the invariance of the action under $\tau$ and $\vec{\sigma}$ parameter changes).

$$
\begin{aligned}
\mathcal{H}_{\mu}(\tau, \vec{\sigma}) & =\rho_{\mu}(\tau, \vec{\sigma})-z_{r \mu}(\tau, \vec{\sigma}) \gamma^{r s}(\tau, \vec{\sigma})\left[\delta^{3}(\vec{\sigma}-\vec{\eta}(\tau)) \kappa_{r}(\tau)+F_{r u}(\tau, \vec{\sigma}) \pi^{u}(\tau, \vec{\sigma})\right]- \\
& -l_{\mu}(\tau, \vec{\sigma})\left[\delta ^ { 3 } ( \vec { \sigma } - \vec { \eta } ( \tau ) ) \left(\sqrt{m^{2} c^{2}+2 m c \Omega \beta^{*}(\tau) \beta(\tau)+\gamma^{r s}(\tau, \vec{\sigma}) \kappa_{r}(\tau) \kappa_{s}(\tau)}+\right.\right. \\
& -\frac{2\left(m c+\Omega \beta^{*}(\tau) \beta(\tau)\right)}{\sqrt{\gamma(\tau, \vec{\sigma})}} \frac{A^{\mu \nu}(\tau)\left(l_{\mu} z_{s \nu} \pi^{s}\right)(\tau, \vec{\sigma})}{\sqrt{m^{2} c^{2}+2 m c \Omega \beta^{*}(\tau) \beta(\tau)+\gamma^{r s}(\tau, \vec{\sigma}) \kappa_{r}(\tau) \kappa_{s}(\tau)}}+ \\
& \left.+\left(m c+\Omega \beta^{*}(\tau) \beta(\tau)\right) \frac{A^{\mu \nu}(\tau)\left(z_{u \mu} z_{v \nu} \gamma^{u r} \gamma^{v s} F_{r s}\right)(\tau, \vec{\sigma})}{\sqrt{m^{2} c^{2}+\Omega \beta^{*}(\tau) \beta(\tau)+\gamma^{r s}(\tau, \vec{\sigma}) \kappa_{r}(\tau) \kappa_{s}(\tau)}}\right)+ \\
& \left.+\left(-\frac{1}{2 \sqrt{\gamma}}{ }^{4} g_{r s} \pi^{r} \pi^{s}+\frac{\sqrt{\gamma}}{4} \gamma^{r s} \gamma^{u v} F_{r u} F_{s v}\right)(\tau, \vec{\sigma})\right]= \\
& =\rho_{\mu}(\tau, \vec{\sigma})-\left(\sqrt{\gamma}\left[l_{\mu} T_{\perp \perp}-z_{r \mu} \gamma^{r s} T_{\perp s}\right]\right)(\tau, \vec{\sigma}) \approx 0
\end{aligned}
$$

where $T_{\perp \perp}=l_{\mu} l_{\nu} T^{\mu \nu}$ and $T_{\perp r}=l_{\mu} z_{r \nu} T^{\mu \nu}$ are components of the energy momentum tensor of the isolated system(see Section III of Ref.[10]). 
The Poisson brackets of the non-Grassmann variables are: $\left\{z^{\mu}(\tau, \vec{\sigma}), \rho_{\nu}\left(\tau, \vec{\sigma}_{1}\right)\right\}=$ $-\delta_{\nu}^{\mu} \delta^{3}\left(\vec{\sigma}-\vec{\sigma}_{1}\right),\left\{A_{A}(\tau, \vec{\sigma}), \pi^{B}\left(\tau, \vec{\sigma}_{1}\right)\right\}=\delta_{A}^{B} \delta^{3}\left(\vec{\sigma}-\vec{\sigma}_{1}\right),\left\{\eta^{r}(\tau), \kappa_{s}(\tau)\right\}=-\delta_{s}^{r},\left\{\lambda_{1}(\tau), \pi_{\lambda_{1}}(\tau)\right\}=$ 1 .

The Dirac Hamiltonian containing the canonical Hamiltonian (2.9) and the primary constraints is

$$
\begin{aligned}
H_{D} & =-\lambda_{1}(\tau)\left(\alpha^{*}(\tau) \alpha(\tau)+\beta^{*}(\tau) \beta(\tau)\right)+\gamma_{1}(\tau) \pi_{\lambda_{1}}(\tau)+ \\
& +\zeta_{\mu}(\tau) \chi^{\mu}(\tau)+\zeta_{(\alpha)}(\tau) \chi_{(\alpha)}(\tau)+\zeta_{(\beta)}(\tau) \chi_{(\beta)}(\tau)+\zeta_{(\alpha)}^{*}(\tau) \chi_{(\alpha)}^{*}(\tau)+\zeta_{(\beta)}^{*}(\tau) \chi_{(\beta)}^{*}(\tau)+ \\
& +\int d^{3} \sigma\left[\lambda^{\mu}(\tau, \vec{\sigma}) \mathcal{H}_{\mu}(\tau, \vec{\sigma})+\lambda_{\tau}(\tau, \vec{\sigma}) \pi^{\tau}(\tau, \vec{\sigma})-A_{\tau}(\tau, \vec{\sigma}) \partial_{r} \pi^{r}(\tau, \vec{\sigma})\right]
\end{aligned}
$$

where $\gamma_{1}(\tau), \zeta_{\mu}(\tau), \zeta_{(\alpha)}(\tau), \zeta_{(\beta)}(\tau), \zeta_{(\alpha)}^{*}(\tau), \zeta_{(\beta)}^{*}(\tau), \lambda^{\mu}(\tau, \vec{\sigma}), \lambda_{\tau}(\tau, \vec{\sigma})$, are Dirac multipliers.

The preservation in time of the primary constraints implies the following secondary constraints

$$
\begin{aligned}
& \alpha^{*}(\tau) \alpha(\tau)+\beta^{*}(\tau) \beta(\tau) \approx 0, \\
& \Gamma(\tau, \vec{\sigma})=\partial_{r} \pi^{r}(\tau, \vec{\sigma}) \approx 0 .
\end{aligned}
$$

While the Grassmann constraints (3.1) are second class, all the other constraints are first class ${ }^{5}$ and the variables $\lambda_{1}(\tau), z^{\mu}(\tau, \vec{\sigma}), A_{\tau}(\tau, \vec{\sigma})$ and the longitudinal component of the vector potential are gauge variables.

As shown in Ref.[12] we can eliminate the second class Grassmann constraints (3.1) by replacing the Poisson brackets $\left\{\xi^{\mu}(\tau), \pi_{\xi}^{\nu}(\tau)\right\}=-\eta^{\mu \nu},\left\{\alpha(\tau), \pi_{\alpha}(\tau)\right\}=\left\{\alpha^{*}(\tau), \pi_{\alpha}^{*}(\tau)\right\}=$ $\left\{\beta(\tau), \pi_{\beta}(\tau)\right\}=\left\{\beta^{*}(\tau), \pi_{\beta}^{*}(\tau)\right\}=-1$ with the following Dirac brackets (still denoted $\{.,$. for the sake of simplicity)

$$
\begin{aligned}
& \left\{\xi^{\mu}(\tau), \xi^{\nu}(\tau)\right\}=-i \eta^{\mu \nu}, \\
& \left\{\alpha(\tau), \alpha^{*}(\tau)\right\}=\left\{\beta(\tau), \beta^{*}(\tau)\right\}=-i
\end{aligned}
$$

Then the Dirac Hamiltonian becomes

$$
\begin{aligned}
H_{D} & =-\lambda_{1}(\tau)\left(\alpha^{*}(\tau) \alpha(\tau)+\beta^{*}(\tau) \beta(\tau)\right)+\gamma_{1}(\tau) \pi_{\lambda_{1}}(\tau)+ \\
& +\int d^{3} \sigma\left[\lambda^{\mu}(\tau, \vec{\sigma}) \mathcal{H}_{\mu}(\tau, \vec{\sigma})+\lambda_{\tau}(\tau, \vec{\sigma}) \pi^{\tau}(\tau, \vec{\sigma})-A_{\tau}(\tau, \vec{\sigma}) \partial_{r} \pi^{r}(\tau, \vec{\sigma})\right]
\end{aligned}
$$

\footnotetext{
${ }^{5}$ As in Ref.[12] this is a non trivial check. What turns out to be first class are not the constraints $\mathcal{H}_{\mu}(\tau, \vec{\sigma}) \approx 0$, but modified constraints obtained by adding to $\mathcal{H}_{\mu}(\tau, \vec{\sigma})$ linear combinations of the Grassmann constraints $\chi^{\mu}(\tau) \approx 0$ of Eqs.(3.1). Since the calculations are the same given in Ref.[12], we do not reproduce them.
} 


\section{A. The Transversality Constraint} $\xi^{\mu}(\tau)$

At this point we add by hand the transversality constraints on the Grassmann variables

$$
\Phi(\tau)=P_{\mu} \xi^{\mu}(\tau) \approx 0
$$

with the conserved total $P^{\mu}$ given in Eq.(2.10). This constraint is second class and eliminates the time-like component of $\xi_{\tau}^{\mu}$, so that there is only an independent Wigner spin-1 Grassmann 3 -vector

$$
\xi_{\perp}^{r}(\tau)=\epsilon_{\mu}^{r}(\vec{h}) \xi^{\mu}(\tau) .
$$

If we go to Dirac brackets by eliminating the second class constraint (3.8) (so that $\xi^{\mu}(\tau) \equiv$ $\left.\epsilon_{r}^{\mu}(\vec{h}) \xi_{\perp}^{r}(\tau)\right)$, we get

$$
\left\{\xi_{\perp}^{r}(\tau), \xi_{\perp}^{s}(\tau)\right\}^{*}=i \delta^{r s}
$$

with all the other basic Poisson brackets left unmodified except the following ones

$$
\left\{z^{\mu}(\tau, \vec{\sigma}), z^{\nu}\left(\tau, \vec{\sigma}_{1}\right)\right\}^{*} \neq 0, \quad\left\{z^{\mu}(\tau, \vec{\sigma}), \xi_{\perp}^{r}(\tau)\right\}^{*} \neq 0
$$

The new canonical variable $\tilde{z}^{\mu}(\tau, \vec{\sigma})$ satisfying the standard Poisson brackets with all the other variables is

$$
\tilde{z}^{\mu}(\tau, \vec{\sigma})=z^{\mu}(\tau, \vec{\sigma})+\frac{i}{2} \epsilon_{\nu}^{A}(\vec{h}) \eta_{A B} \frac{\partial \epsilon_{\rho}^{B}(\vec{h})}{\partial P_{\mu}} \epsilon_{r}^{\rho}(\vec{h}) \epsilon_{s}^{\nu}(\vec{h}) \xi_{\perp}^{r}(\tau) \xi_{\perp}^{s}(\tau)
$$

As a consequence the Lorentz generators of Eqs.(2.10) become

$$
\begin{gathered}
J^{\mu \nu}=\int d^{3} \sigma\left[\tilde{z}^{\mu}(\tau, \vec{\sigma}) \rho^{\nu}(\tau, \vec{\sigma})-\tilde{z}^{\nu}(\tau, \vec{\sigma}) \rho^{\mu}(\tau, \vec{\sigma})\right]+\tilde{S}_{\xi}^{\mu \nu} \\
\tilde{S}_{\xi}^{i j}=\epsilon^{i j r} S_{\xi}^{r}, \quad S_{\xi}^{r}=-\frac{i}{2} \epsilon^{r u v} \xi_{\perp}^{u} \xi_{\perp}^{v} \\
\tilde{S}_{\xi}^{o i}=-\frac{\epsilon^{i j r} P^{j} S_{\xi}^{r}}{P^{o}+\sqrt{\epsilon P^{2}}} .
\end{gathered}
$$

\section{B. The Restriction to the Wigner 3-spaces of the Inertial Rest Frame}

As shown in Refs. [5, 10] the restriction to the inertial rest frame is obtained by adding the gauge fixing $z^{\mu}(\tau, \vec{\sigma}) \approx z_{(W)}^{\mu}(\tau, \vec{\sigma})$ with the embedding of Eq.(1.1), in which $x_{o}^{\mu}$ is identified with $Y^{\mu}(0)$ so that the observer, origin of the 3-coordinates on the Wigner 3-spaces, becomes the Fokker-Pryce center of inertia, i.e. $Y^{\mu}(\tau)=z_{W}^{\mu}(\tau, 0)=Y^{\mu}(0)+h^{\mu} \tau\left(h^{\mu}=P^{\mu} / \sqrt{\epsilon P^{2}}=\right.$ $l^{\mu}$ is the unit normal to Wigner 3-spaces). Then Eq.(3.12) becomes 


$$
\begin{aligned}
\tilde{z}^{\mu}(\tau, \vec{\sigma}) & \approx Y^{\mu}(\tau)+\epsilon_{r}^{\mu}(\vec{h}) \sigma^{r}+\frac{i}{2} \epsilon_{\nu}^{A}(\vec{h}) \eta_{A B} \frac{\partial \epsilon_{\rho}^{B}(\vec{h})}{\partial P_{\mu}} \epsilon_{r}^{\rho}(\vec{h}) \epsilon_{s}^{\nu}(\vec{h}) \xi_{\perp}^{r}(\tau) \xi_{\perp}^{s}(\tau)= \\
& \stackrel{\text { def }}{=} \hat{x}^{\mu}(\tau)+\epsilon_{r}^{\mu}(\vec{h}) \sigma^{r} .
\end{aligned}
$$

The new position $\hat{x}^{\mu}(\tau)$ allows the use Eqs.(3.13) to define the following spin-like tensor $S_{s}^{\mu \nu}=J^{\mu \nu}-\left(\hat{x}^{\mu}(\tau) P^{\nu}-\hat{x}^{\nu}(\tau) P^{\mu}\right)$.

As shown in Ref.[10], if we evaluate the Poincaré generators (3.13) with the embed$\operatorname{ding} z_{(W)}^{\mu}(\tau, \vec{\sigma})$, we get the following description of the isolated system "atom plus electromagnetic field":

1) There is a decoupled (non-local) non-covariant canonical external center of mass $\tilde{x}^{\mu}(\tau)$ whose conjugate canonical momentum is $P^{\mu}=M c h^{\mu}\left(M c=\sqrt{\epsilon P^{2}}\right.$ is the invariant mass of the isolated system)

$$
\begin{aligned}
\tilde{x}^{\mu}(\tau)= & Y^{\mu}(\tau)+\frac{i}{2} \epsilon_{\nu}^{A}(\vec{h}) \eta_{A B} \frac{\partial \epsilon_{\rho}^{B}(\vec{h})}{\partial P_{\mu}} \epsilon_{r}^{\rho}(\vec{h}) \epsilon_{s}^{\nu}(\vec{h}) \xi_{\perp}^{r}(\tau) \xi_{\perp}^{s}(\tau)- \\
- & \epsilon_{\nu}^{A}(\vec{h}) \eta_{A B} \frac{\partial \epsilon_{\rho}^{B}(\vec{h})}{\partial P_{\mu}} S_{s}^{\mu \nu}, \\
& \left\{\tilde{x}^{\mu}(\tau), P^{\nu}\right\}^{*}=-\eta^{\mu \nu}, \quad\left\{\tilde{x}^{\mu}(\tau), \tilde{x}^{\nu}(\tau)\right\}^{*}=0 .
\end{aligned}
$$

This decoupled non-covariant point particle carries a pole-dipole structure (the invariant mass $M$ and the rest spin $\vec{S}$ of the isolated system) and an external realization of the Poincaré generators ${ }^{6}$

$$
\begin{aligned}
P^{\mu}= & M c h^{\mu}, \quad J^{\mu \nu}=\tilde{x}^{\mu} P^{\nu}-\tilde{x}^{\nu} P^{\mu}+\tilde{S}^{\mu \nu} \\
& \tilde{S}^{\mu \nu}=\tilde{S}_{s}^{\mu \nu}+\tilde{S}_{\xi}^{\mu \nu} \\
& \tilde{S}^{o i}=\tilde{S}_{s}^{o i}=-\frac{\epsilon^{i j r} h^{j} S^{r}}{1+\sqrt{1+\vec{h}^{2}}} \\
& \tilde{S}^{i j}=\tilde{S}_{s}^{i j}+\tilde{S}_{\xi}^{i j}=\epsilon^{i j r} S^{r} .
\end{aligned}
$$

2) Inside the Wigner 3-spaces of the rest frame the system "atom plus electro-magnetic field" is described by the atom canonical 3-coordinates $\vec{\eta}(\tau), \vec{\kappa}(\tau)$ (plus the Grassmann

${ }^{6}$ As shown in Refs. $[5,10,16]$ the non-covariant canonical center of mass $\tilde{x}^{\mu}(\tau)$ and its momentum $P^{\mu}$ can be replaced with the frozen Jacobi data $\vec{z}, \vec{h},\left\{z^{i}, h^{j}\right\}=\delta^{i j}$, by means of the expressions $\tilde{x}^{\mu}(\tau)=$ $\left(\sqrt{1+\vec{h}^{2}}\left[\tau+\frac{\vec{h} \cdot \vec{z}}{M c}\right] ; \frac{\vec{z}}{M c}+\left(\tau+\frac{\vec{h} \cdot \vec{z}}{M c}\right) \vec{h}\right), P^{\mu}=M c h^{\mu}=M c\left(\sqrt{1+\vec{h}^{2}} ; \vec{h}\right)$. The Cauchy data for the NewtonWigner position are $\vec{z} / M c$. Then we get that $L^{\mu \nu}=\tilde{x}^{\mu} P^{\nu}-\tilde{x}^{\nu} P^{\mu}$ has the components $L^{o i}=-\sqrt{1+\vec{h}^{2}} z^{i}$ and $L^{i j}=z^{i} h^{j}-z^{j} h^{i}$. 
variables $\left.\xi_{\perp}^{r}(\tau), \alpha(\tau), \alpha^{*}(\tau), \beta(\tau), \beta^{*}(\tau)\right)$ and by the canonical coordinates $A_{A}(\tau, \vec{\sigma}), \pi^{A}(\tau, \vec{\sigma})$ of the electro-magnetic field. There is a unfaithful internal representation representation $M c, \mathcal{P}^{r}, S^{r}, \mathcal{K}^{r}$, of the Poincaré generators restricted by the conditions $\mathcal{P}^{r} \approx 0$ and $\mathcal{K}^{r} \approx 0$ (they are the rest-frame conditions eliminating the internal center of mass inside the Wigner 3-space). These generators have the following expression

$$
\begin{aligned}
M c & =\sqrt{m^{2} c^{2}+2 m c \Omega \beta^{*}(\tau) \beta(\tau)+\vec{\kappa}^{2}(\tau)}+ \\
& +\frac{m c}{\sqrt{m^{2} c^{2}+\vec{\kappa}^{2}(\tau)}}\left(\beta^{*}(\tau) \alpha(\tau)+\alpha^{*}(\tau) \beta(\tau)\right) \vec{d}(\tau) \cdot \vec{\pi}(\tau, \vec{\eta}(\tau))+ \\
& +\frac{1}{2} \int d^{3} \sigma\left(\vec{\pi}^{2}(\tau, \vec{\sigma})+\vec{B}^{2}(\tau, \vec{\sigma})\right), \\
\vec{S} & =\vec{\eta}(\tau) \times \vec{\kappa}(\tau)-\frac{i}{2} \vec{\xi}_{\perp}(\tau) \times \vec{\xi}_{\perp}(\tau)+\int d^{3} \sigma \vec{\sigma} \times(\vec{\pi}(\tau, \vec{\sigma}) \times \vec{B}(\tau, \vec{\sigma})), \\
\overrightarrow{\mathcal{P}} & =\vec{\kappa}(\tau)+\int d^{3} \sigma \vec{\pi}(\tau, \vec{\sigma}) \times \vec{B}(\tau, \vec{\sigma}) \approx 0, \\
\overrightarrow{\mathcal{K}} & =-\vec{\eta}(\tau)\left(\sqrt{m^{2} c^{2}+2 m c \Omega \beta^{*}(\tau) \beta(\tau)+\vec{\kappa}^{2}(\tau)}+\right. \\
& \left.+\frac{m c}{\sqrt{m^{2} c^{2}+\vec{\kappa}^{2}(\tau)}}\left(\beta^{*}(\tau) \alpha(\tau)+\alpha^{*}(\tau) \beta(\tau)\right) \vec{d}(\tau) \cdot \vec{\pi}(\tau, \vec{\eta}(\tau))\right)- \\
& -\frac{1}{2 c} \int d^{3} \sigma \vec{\sigma}\left(\vec{\pi}^{2}(\tau, \vec{\sigma})+\vec{B}^{2}(\tau, \vec{\sigma})\right) \approx 0 .
\end{aligned}
$$

The invariant mass $M c=\sqrt{\epsilon P^{2}}$ is derived from Eqs. (2.10) and (3.3) restricted to $z_{(W)}^{\mu}(\tau, \vec{\sigma})$ by noting that Eq. $(2.6)$ and $z_{(W) A}^{\mu}(\tau, \vec{\sigma})=\epsilon_{A}^{\mu}(\vec{h})$ imply $A^{\mu \nu}(\tau) \epsilon_{u \mu}(\vec{h}) \epsilon_{v \nu}(\vec{h})=0$ and $A^{\mu \nu}(\tau) h_{\mu} \epsilon_{s \nu}(\vec{h})=\left(\beta^{*}(\tau) \alpha(\tau)+\alpha^{*}(\tau) \beta(\tau)\right) d^{s}(\tau)$, where we introduced the electric dipole

$$
\vec{d}(\tau)=-i d \vec{\xi}_{\perp}(\tau) \times \vec{\xi}_{\perp}(\tau)
$$

The non-relativistic limit of the atom energy is $M c^{2}=m c^{2}+\frac{\vec{k}^{2}(\tau)}{2 m}+\frac{\Omega}{m} \beta^{*}(\tau) \beta(\tau)+$ $\left(\beta^{*}(\tau) \alpha(\tau)+\alpha^{*}(\tau) \beta(\tau)\right) c \vec{d}(\tau) \cdot \vec{\pi}(\tau, \vec{\eta}(\tau))$. After a quantization done with the method explained in the next Section it reproduces the Hamiltonian of the Rabi model $[1,3,4]$ with $\tilde{\Omega}=\Omega / m$ and electric dipole $c \vec{d}(\tau)$. By making the rotating phase approximation one finds the Hamiltonian of the Jaynes-Cummings model [2-4].

As shown in Refs. $[5,10]$ the Dirac Hamiltonian in the rest frame is

$$
\begin{aligned}
H_{D} & =M c-\lambda_{1}(\tau)\left(\alpha^{*}(\tau) \alpha(\tau)+\beta^{*}(\tau) \beta(\tau)\right)+\gamma_{1}(\tau) \pi_{\lambda_{1}}(\tau)+ \\
& +\int d^{3} \sigma\left[\lambda_{\tau}(\tau, \vec{\sigma}) \pi^{\tau}(\tau, \vec{\sigma})-A_{\tau}(\tau, \vec{\sigma}) \partial_{r} \pi^{r}(\tau, \vec{\sigma})\right]
\end{aligned}
$$

To the resulting Hamilton equations one must add the rest-frame constraints $\overrightarrow{\mathcal{P}} \approx 0$ and $\overrightarrow{\mathcal{K}} \approx 0$. With the gauge fixing $\lambda_{1}(\tau) \approx 0$ we can also eliminate the gauge variable $\lambda_{1}(\tau):$ 
the Dirac Hamiltonian reduces to $H_{D}=M c+\int d^{3} \sigma\left[\lambda_{\tau}(\tau, \vec{\sigma}) \pi^{\tau}(\tau, \vec{\sigma})-A_{\tau}(\tau, \vec{\sigma}) \partial_{r} \pi^{r}(\tau, \vec{\sigma})\right]$ and we have to add the constraint $\alpha^{*}(\tau) \alpha(\tau)+\beta^{*}(\tau) \beta(\tau) \approx 0$ to the Hamilton equations.

\section{The Restriction to the Radiation Gauge}

In Refs. $[5,10]$ it is shown how to make the restriction to the radiation gauge, where the electro-magnetic field is described by the transverse quantities $\vec{A}_{\perp}(\tau, \vec{\sigma})$ and $\vec{\pi}_{\perp}(\tau, \vec{\sigma})$. In the rest frame we have $\vec{\pi}_{\perp}(\tau, \vec{\sigma})=\vec{E}_{\perp}(\tau, \vec{\sigma})$ and $\vec{B}(\tau, \vec{\sigma})=\vec{\partial} \times \vec{A}_{\perp}(\tau, \vec{\sigma})$. All the previous formulas remain valid with the replacement $\vec{\pi} \mapsto \vec{\pi}_{\perp}$. Now the Dirac Hamiltonian (3.19) is $H_{D}=M c$.

As shown in Ref.[5] we have the following representation of the electro-magnetic fields in the radiation gauge

$$
\begin{aligned}
& \overrightarrow{\tilde{A}}_{\perp}(\tau, \vec{k})=\frac{i}{\vec{k}^{2}} \vec{k} \times \overrightarrow{\tilde{B}}(\tau, \vec{k})=\frac{1}{2|\vec{k}|}\left[\vec{\alpha}(\tau, \vec{k})+\vec{\alpha}^{*}(\tau,-\vec{k})\right], \\
& \vec{A}_{\perp}(\tau, \vec{\sigma})=\frac{1}{(2 \pi)^{3}} \int \frac{d^{3} k}{2 \omega(\vec{k})} \sum_{\lambda=1,2} \vec{\epsilon}_{\lambda}(\vec{k})\left[a_{e m \lambda}(\tau, \vec{k}) e^{i \vec{k} \cdot \vec{\sigma}}+a_{e m \lambda}^{*}(\tau, \vec{k}) e^{-i \vec{k} \cdot \vec{\sigma}}\right], \\
& \vec{\pi}_{\perp}(\tau, \vec{\sigma})=\frac{i}{2(2 \pi)^{3}} \int d^{3} k \sum_{\lambda=1,2} \vec{\epsilon}_{\lambda}(\vec{k})\left[a_{e m \lambda}(\tau, \vec{k}) e^{i \vec{k} \cdot \vec{\sigma}}-a_{e m \lambda}^{*}(\tau, \vec{k}) e^{-i \vec{k} \cdot \vec{\sigma}}\right]= \\
& \stackrel{\circ}{=}_{d y n}-\frac{\partial \vec{A}_{\perp}(\tau, \vec{\sigma})}{\partial \tau} \\
& \vec{B}(\tau, \vec{\sigma})=\frac{i}{2(2 \pi)^{3}} \int \frac{d^{3} k}{\omega(\vec{k})} \vec{k} \times \sum_{\lambda=1,2} \vec{\epsilon}_{\lambda}(\vec{k})\left[a_{e m \lambda}(\tau, \vec{k}) e^{i \vec{k} \cdot \vec{\sigma}}-a_{e m \lambda}^{*}(\tau, \vec{k}) e^{-i \vec{k} \cdot \vec{\sigma}}\right], \\
& a_{e m \lambda}(\tau, \vec{k})=\int d^{3} \sigma \vec{\epsilon}_{\lambda}(\vec{k}) \cdot\left[\omega(\vec{k}) \vec{A}_{\perp}(\tau, \vec{\sigma})-i \vec{\pi}_{\perp}(\tau, \vec{\sigma})\right] e^{-i \vec{k} \cdot \vec{\sigma}} \\
& \left\{a_{e m \lambda}(\tau, \vec{k}), a_{e m \lambda^{\prime}}^{*}\left(\tau, \vec{k}^{\prime}\right)\right\}=-i \Omega(\vec{k}) c \delta_{\lambda \lambda^{\prime}} \delta^{3}\left(\vec{k}-\vec{k}^{\prime}\right) .
\end{aligned}
$$




\section{THE QUANTIZATION}

In Refs.[5, 6] we presented the classical theory underlying relativistic atomic physics. In Ref.[15] we developed a new version of relativistic quantum mechanics in the inertial rest-frame instant form consistent with what is known about relativistic bound states and taking into account the non-covariance of the canonical relativistic external center of mass. It can be used to quantize the atoms in the absence of the electro-magnetic field. The main complication is the imposition of the rest-frame conditions $\overrightarrow{\mathcal{P}} \approx 0$ and $\overrightarrow{\mathcal{K}} \approx 0$ due to the complicated form of the boost generators in presence of interactions among the particles. When they cannot be solved at the classical level (which would allow quantization of only physical degrees of freedom), one must quantize all the canonical variables in the Wigner 3-space in a un-physical Hilbert space and then select the physical states by asking that they satisfy $<\Phi_{\text {phys }}|\hat{\overrightarrow{\mathcal{P}}}| \Phi_{\text {phys }}>=0$ and $<\Phi_{\text {phys }}|\hat{\overrightarrow{\mathcal{K}}}| \Phi_{\text {phys }}>=0$. This type of quantization has not yet been done for the free transverse electro-magnetic field in the radiation gauge much less for the system of charged particles plus a generic transverse electro-magnetic field.

Therefore we will give a quantization of the atom in an external classical transverse electro-magnetic field and then we will show its coupling to a single mode of a free field (this is suitable for a two-level atom) as it is often done in atomic physics [3].

The canonical 3-coordinates $\vec{\eta}(\tau), \vec{\kappa}(\tau)$, are quantized in the standard way: in the coordinate representation we have $\vec{\eta} \mapsto \vec{\eta}$ and $\vec{\kappa} \mapsto-i \hbar \frac{\partial}{\partial \vec{\eta}}$ as unbounded operators in a Hilbert space with the standard scalar product.

The Grassmann variables $\xi_{\perp}^{r}$ describing the electric dipole are quantized to the Pauli matrices, $\xi_{\perp}^{r} \mapsto \sqrt{\frac{\hbar}{2}} \sigma^{r}$ like for the spinning particle of Ref.[12]. Therefore, Eqs.(3.18) gives a quantum electric dipole $\hat{\vec{d}}$ behaving like a spin $1 / 2$ Pauli particle.

Each pair of complex Grassmann variables $\alpha, \alpha^{*}$, and $\beta, \beta^{*}$ is quantized to operators $\hat{a}, \hat{a}^{\dagger}$, and $\hat{b}, \hat{b}^{\dagger}$ corresponding to Fermi oscillators, namely satisfying the anti-commutation relations $\left[\hat{a}, \hat{a}^{\dagger}\right]_{+}=\left[\hat{b}, \hat{b}^{\dagger}\right]_{+}=\hbar$ and with one Fermi oscillator commuting with the other one. Therefore we get a 4-dimensional Hilbert space whose states have the form

$$
\begin{aligned}
\Psi= & C_{++} \Psi_{\alpha}(+) \otimes \Psi_{\beta}(+)+C_{-+} \Psi_{\alpha}(-) \otimes \Psi_{\beta}(+)+ \\
+ & C_{+-} \Psi_{\alpha}(+) \otimes \Psi_{\beta}(-)+C_{--} \Psi_{\alpha}(-) \otimes \Psi_{\beta}(-), \\
& \hat{a} \Psi=C_{-+} \Psi_{\alpha}(+) \otimes \Psi_{\beta}(+)+C_{--} \Psi_{\alpha}(+) \otimes \Psi_{\beta}(-), \\
& \hat{a}^{\dagger} \Psi=C_{++} \Psi_{\alpha}(-) \otimes \Psi_{\beta}(+)+C_{+-} \Psi_{\alpha}(-) \otimes \Psi_{\beta}(-), \\
& \hat{b} \Psi=C_{++} \Psi_{\alpha}(+) \otimes \Psi_{\beta}(-)+C_{-+} \Psi_{\alpha}(-) \otimes \Psi_{\beta}(-), \\
& \hat{b}^{\dagger} \Psi=C_{+-} \Psi_{\alpha}(+) \otimes \Psi_{\beta}(+)+C_{--} \Psi_{\alpha}(-) \otimes \Psi_{\beta}(+),
\end{aligned}
$$

If we choose the following ordering 


$$
\begin{aligned}
& \alpha^{*} \alpha \mapsto-\hat{a}^{\dagger} \hat{a}+\frac{\hbar}{2} \\
& \beta^{*} \beta \mapsto \hat{b}^{\dagger} \hat{b}-\frac{\hbar}{2}
\end{aligned}
$$

then the constraint $\alpha^{*}(\tau) \alpha(\tau)+\beta^{*}(\tau) \beta(\tau) \approx 0$ implies the following condition on the physical states

$$
\begin{aligned}
& \left(\hat{b}^{\dagger} \hat{b}-\hat{a}^{\dagger} \hat{a}\right) \Psi_{\text {phys }}=0 \\
& \Downarrow \\
& \Psi_{\text {phys }}=C_{-+} \Psi_{\alpha}(-) \otimes \Psi_{\beta}(+)+C_{+-} \Psi_{\alpha}(+) \otimes \Psi_{\beta}(-)= \\
& \stackrel{\text { def }}{=} C_{-+} \Phi(+)+C_{+-} \Phi(-) \text {. }
\end{aligned}
$$

If we define the operators

$$
\hat{c}=\hat{b}^{\dagger} \hat{a}, \quad \hat{c}^{\dagger}=\hat{b} \hat{a}^{\dagger},
$$

we get

$$
\begin{aligned}
& \hat{c} \Phi(+)=\Phi(-), \quad \hat{c} \Phi(-)=0, \\
& \hat{c}^{\dagger} \Phi(-)=\Phi(+), \quad \hat{c}^{\dagger} \Phi(+)=0, \\
& \hat{b}^{\dagger} \hat{b} \Psi_{\text {phys }}=\hat{c}^{\dagger} \hat{c} \Psi_{\text {phys }} .
\end{aligned}
$$

Therefore the mass term $m c+\Omega \beta^{*} \beta$ will become the mass $m c+\Omega \hat{c}^{\dagger} \hat{c}$ of the two physical levels.

Moreover Eq.(4.4) implies that the coupling term of the electric dipole to the electric field present in $M c$ of Eq.(3.17) takes the form

$$
\frac{m c}{\sqrt{m^{2} c^{2}+\hat{\vec{\kappa}}^{2}}}\left(\hat{c}^{\dagger}+\hat{c}\right) \hat{\vec{d}} \cdot \vec{E}(\tau, \vec{\eta}(\tau)) .
$$

As is usually done in atomic physics, in particular in the treatment of two-level atoms $[3,4]$, let us consider only one quantized mode with energy $\hbar \omega\left(\hat{a}_{e m}^{\dagger} \hat{a}_{e m}+\frac{1}{2}\right)$ of the transverse electro-magnetic field (3.20). Then the transverse electric field can be approximated as $\overrightarrow{\mathcal{E}}(\tau)\left(\hat{a}_{e m}+\hat{a}_{e m}^{\dagger}\right)$.

Since in the physical Hilbert space the operators $\hat{c}^{\dagger} \hat{c}, \hat{c}^{\dagger}$ and $\hat{c}$ coincide with the Pauli matrices $\sigma_{3}, \sigma_{+}$and $\sigma_{-}$respectively, in the radiation gauge the quantum Dirac Hamiltonian takes the following form 


$$
\begin{aligned}
\hat{H}_{D} & =\sqrt{m^{2} c^{2}+2 m c \Omega \sigma_{3}+\hat{\vec{k}}^{2}}+\hbar \omega\left(\hat{a}_{e m}^{\dagger} \hat{a}_{e m}+\frac{1}{2}\right)+ \\
& +\frac{m c}{\sqrt{m^{2} c^{2}+\hat{\vec{k}}^{2}}}(\hat{\vec{d}} \cdot \overrightarrow{\mathcal{E}}(\tau))\left(\hat{c}^{\dagger}+\hat{c}\right)\left(\hat{a}_{e m}+\hat{a}_{e m}^{\dagger}\right),
\end{aligned}
$$

whose non-relativistic limit is $(\tilde{\Omega}=\Omega / m)$

$$
\begin{aligned}
\hat{H} & =\frac{\hat{\vec{\kappa}}^{2}}{2 m}+\frac{\hbar}{2} \tilde{\Omega} \sigma_{3}+\hbar \omega\left(\hat{a}_{e m}^{\dagger} \hat{a}_{e m}+\frac{1}{2}\right)+ \\
& +(c \hat{\vec{d}} \cdot \overrightarrow{\mathcal{E}}(\tau))\left(\sigma_{+}+\sigma_{-}\right)\left(\hat{a}_{e m}+\hat{a}_{e m}^{\dagger}\right) .
\end{aligned}
$$

But this is this is the Hamiltonian of the Rabi model $[1,3,4]$, which becomes the JaynesCummings one [2-4] in the rotating phase approximation, in which the last term becomes $(c \hat{\vec{d}} \cdot \overrightarrow{\mathcal{E}}(\tau))\left(\sigma_{+} \hat{a}_{e m}+\sigma_{-} \hat{a}_{e m}^{\dagger}\right)$.

In the rest frame description one should add the rest-frame conditions < $\Phi_{\text {phys }}|\hat{\overrightarrow{\mathcal{P}}}| \Phi_{\text {phys }}>=0$ and $<\Phi_{\text {phys }}|\hat{\overrightarrow{\mathcal{K}}}| \Phi_{\text {phys }}>=0$ in the same approximation. 


\section{CONCLUSIONS}

Our model for the neutral two-level atom allowed us to get the relativistic generalization of the Hamiltonians of the Rabi and Jaynes-Cummings models. Moreover we found the explicit form of the Poincaré generators. We also showed how one could describe a charged two-level atom with also a magnetic dipole. These relativistic models could be applied in future use of two-level atoms in space experiments near the Earth, where atomic physics must take into account both special relativity and post-Newtonian general relativity.

To arrive at these results we had to describe the isolated system atom plus dynamical electro-magnetic field as a parametrized Minkowski theory. Instead usually in atomic physics one considers the two-level atom interacting with an external electro-magnetic field in the absolute Euclidean 3-space of Galilei space-time identified with the Euclidean 3-space of a relativistic inertial frame. But this is relativistically problematic because interaction terms like $\vec{d} \cdot \vec{E}$ should be interpreted in the inertial frame instantaneously comoving with the atom, which has an accelerated motion in 3-space. Moreover the transversality constraint (3.8) is

not well defined if $P^{\mu}$ is the non-conserved momentum of the atom. All these problems are solved by our approach.

Finally let us point out the main open problem in the description of extended atoms. If we have an extended object as an atom with $\mathrm{N}$ constituents, we may describe it as a point-like object by using its multipolar expansion (see Section 5 of Ref.[6]) and replace the equations of motions of the $\mathrm{N}$ constituents with the resulting (often approximate) equations of motions for the multipoles. However we do not have a consistent variational principle containing a point particle (the monopole) carrying all the other multipoles and interacting with the electro-magnetic field so as to get a consistent set of equations of motion (and conservation laws) for the multipoles. The solution of this problem would allow us to describe N-level atoms with arbitrary multipoles. 
[1] I.I.Rabi, On the Process of Space Quantization, Phys.Rev. 49, 324 (1926); Space Quantization in a Gyrating Magnetic Field, 51, 652 (1937).

[2] E.T.Jaynes and F.W.Cummings, Comparison of Quantum and Semi-Classical Radiation Theories with Application to the Beam Maser, Proc. JEEE 51, 89 (1963). B.W.Shore and P.L.Knight, The Jaynes-Cummings Model, J.Mod.Opt. 40, 1195 (1993).

[3] W.P.Schleich, Quantum Optics in Phase Space (Wiley-VCH, Berlin, 2001).

[4] J.Larson, Dynamics of the Jaynes-Cummings and Rabi Models: Old Wine in New Bottles, Phys. Scr. 76, 146 (2007) (quant-ph/0612095).

[5] D.Alba, H.W.Crater and L.Lusanna, Towards Relativistic Atom Physics. I. The Rest-Frame Instant Form of Dynamics and a Canonical Transformation for a system of Charged Particles plus the Electro-Magnetic Field, Canad.J.Phys. 88, 379 (2010) (arXiv: 0806.2383).

[6] D.Alba, H.W.Crater and L.Lusanna, Towards Relativistic Atom Physics. II. Collective and Relative Relativistic Variables for a System of Charged Particles plus the Electro-Magnetic Field, Canad.J.Phys. 88, 425 (2010) (arXiv:0811.0715).

[7] D.Alba, H.W.Crater and L.Lusanna, Hamiltonian Relativistic Two-Body Problem: Center of Mass and Orbit Reconstruction, J.Phys. A40, 9585 (2007) (gr-qc/0610200).

[8] D.Alba, H.W.Crater and L.Lusanna, The Semiclassical Relativistic Darwin Potential for Spinning Particles in the Rest Frame Instant Form: Two-Body Bound States with Spin 1/2 Constituents, Int.J.Mod.Phys. A16, 3365-3478 (2001) (hep-th/0103109).

[9] L.Lusanna, The $N$ - and 1-Time Classical Descriptions of N-Body Relativistic Kinematics and the Electromagnetic Interaction, Int.J.Mod.Phys. A12, 645 (1997).

L.Lusanna, The Chrono-Geometrical Structure of Special and General Relativity: A ReVisitation of Canonical Geometrodynamics, lectures at 42nd Karpacz Winter School of Theoretical Physics: Current Mathematical Topics in Gravitation and Cosmology, Ladek, Poland, 6-11 Feb 2006, Int.J.Geom.Methods in Mod.Phys. 4, 79 (2007). (gr-qc/0604120).

[10] D.Alba and L.Lusanna, Charged Particles and the Electro-Magnetic Field in Non-Inertial Frames: I. Admissible 3+1 Splittings of Minkowski Spacetime and the Non-Inertial Rest Frames, Int.J.Geom.Methods in Physics 7, 33 (2010) (0908.0213)

[11] D.Alba and L.Lusanna, Charged Particles and the Electro-Magnetic Field in Non-Inertial Frames: II. Applications: Rotating Frames, Sagnac Effect, Faraday Rotation, Wrap-up Effect , Int.J.Geom.Methods in Physics 7, 185 (2010) (0908.0215).

[12] F.Bigazzi and L.Lusanna, Spinning Particles on Spacelike Hypersurfaces and their Rest Frame Description, Int.J.Mod.Phys. A14, 1429 (1999) (hep-th/9807052).

[13] D.Alba,H.W. Crater and L.Lusanna, Massless Particles plus Matter in the Rest-Frame Instant Form of Dynamics, J.Phys. A 43405203 (arXiv 1005.5521).

[14] D.Alba, H.W.Crater and L.Lusanna, The Rest-Frame Instant Form and Dirac Observables for the Open Nambu String, Eur.Phys.J.Plus 126, 26 (2011) (arXiv 1005.3653).

[15] D.Alba,H.W. Crater and L.Lusanna, Relativistic Quantum Mechanics and Relativistic Entanglement in the Rest-Frame Instant Form of Dynamics, to appear in J.Math.Phys. (arXiv 0907.1816).

[16] D.Alba and L.Lusanna, Quantum Mechanics in Noninertial Frames with a Multitemporal Quantization Scheme: I. Relativistic Particles, Int.J.Mod.Phys. A21, 2781 (2006) (hepth/0502194). 
[17] D.Alba, Quantum Mechanics in Noninertial Frames with a Multitemporal Quantization Scheme: II. Nonrelativistic Particles, Int.J.Mod.Phys. A21, 3917 (hep-th/0504060).

[18] M. O. Scully, and M. S. Zubairy, Quantum optics, (Cambridge, 1997) 